\title{
Optical label encoding using electroabsorption modulators and investigation of chirp properties
}

Xu, Lin; Chi, Nan; Oxenløwe, Leif Katsuo; Yvind, Kresten; Mørk, Jesper; Jeppesen, Palle; Hanberg, Jesper

Published in:

Journal of Lightwave Technology

Link to article, DOI:

10.1109/JLT.2003.815504

Publication date:

2003

Document Version

Publisher's PDF, also known as Version of record

Link back to DTU Orbit

Citation (APA):

Xu, L., Chi, N., Oxenløwe, L. K., Yvind, K., Mørk, J., Jeppesen, P., \& Hanberg, J. (2003). Optical label encoding using electroabsorption modulators and investigation of chirp properties. Journal of Lightwave Technology, 21(8), 1763-1769. https://doi.org/10.1109/JLT.2003.815504

\section{General rights}

Copyright and moral rights for the publications made accessible in the public portal are retained by the authors and/or other copyright owners and it is a condition of accessing publications that users recognise and abide by the legal requirements associated with these rights.

- Users may download and print one copy of any publication from the public portal for the purpose of private study or research.

- You may not further distribute the material or use it for any profit-making activity or commercial gain

- You may freely distribute the URL identifying the publication in the public portal 


\title{
Optical Label Encoding Using Electroabsorption Modulators and Investigation of Chirp Properties
}

\author{
L. Xu, N. Chi, L. K. Oxenløwe, K. Yvind, J. Mørk, P. Jeppesen, Member, IEEE, and J. Hanberg
}

\begin{abstract}
A novel scheme of optical label encoding by wavelength conversion based on electroabsorption modulators (EAMs) is reported. Based on the experimental observations, the chirp properties of the wavelength-converted signal are discussed and a wide dynamic range of the chirp $\alpha$-parameter is found allowed. Compared with cross-gain modulation (XGM) in a semiconductor optical amplifier (SOA), the EAM has several advantages, which make it attractive for optical label encoding or other applications as a wavelength converter.
\end{abstract}

Index Terms-Chirp $\alpha$-parameter, cross-absorption modulation, electroabsorption modulator (EAM), optical label encoding.

\section{INTRODUCTION}

$\mathbf{U}$ LTRA-HIGH speed data-centric networks will likely evolve into an Internet Protocol (IP) network on a wavelength-division-multiplexing (WDM) physical infrastructure [1]. With respect to efficiency and cost effectiveness, new switching technologies are required to route individual packets without converting the packet from optical to electrical format. All-optical packet switching is therefore most likely to be the key technology for the implementation of future IP-over-WDM networks [2]. In addition to the optical wavelength that can serve as an optical label in the multiple protocol wavelength switched (MP $\lambda \mathrm{S}$ ) scheme, a second level of optical label is still necessary for provisioning, maintaining, and restoring switched light-paths. This second level optical label can be realized by subcarrier multiplexing (SCM) [2], [3] or by an orthogonal modulation format [4]-[8] combining amplitude shift keying (ASK) and differential phase shift keying (DPSK) of a single carrier.

Based on the two-level optical label, all-optical processing may be used to realize the wavelength swapping as well as the adding and dropping of the second level label. One promising all-optical processor is the electroabsorption modulator (EAM) [9], [10], which has proven to be a versatile component in ultra fast WDM and OTDM systems with its ability to perform several different functionalities, yet remaining a simple structure. Recently, various all-optical functionalities based on cross-absorption modulation (XAM) [11], [12] have been demonstrated,

Manuscript received January 16, 2003; revised May 13, 2003. This work was supported by the Danish Technical Research Council through the SCOOP project (Semiconductor COmponent for Optical signal Processing) and by the European Union (EU) Commission through the IST project STOLAS (Switching Technologies for Optically Labeled Signals).

L. Xu, N. Chi, L. K. Oxenløwe, K. Yvind, J. Mørk and P. Jeppesen are with Research Center COM, Technical University of Denmark, Lyngby DK-2800, Denmark (e-mail: xl@com.dtu.dk).

J. Hanberg is with the Giga-An Intel Company, Skovlunde DK-2740, Denmark (e-mail: jesper.hanberg@intel.com).

Digital Object Identifier 10.1109/JLT.2003.815504

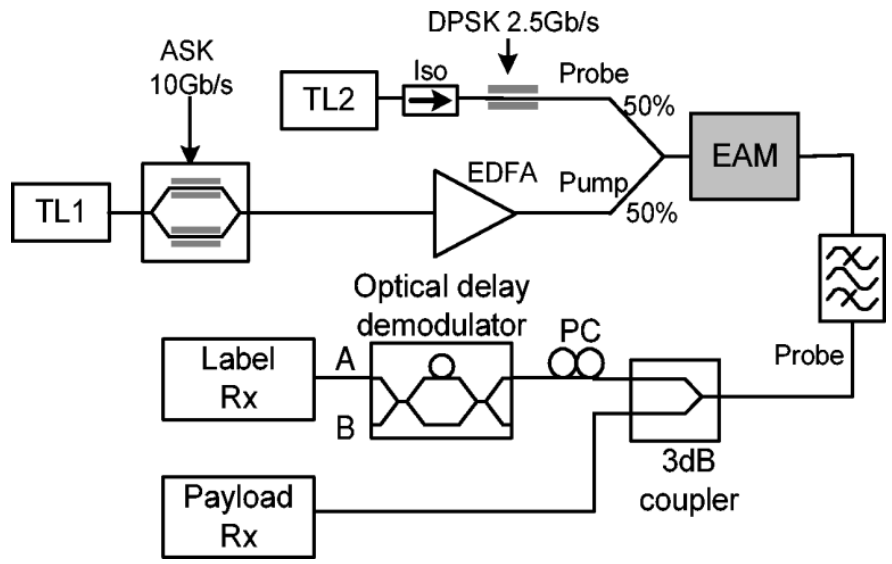

Fig. 1. Experimental setup. TL1: Tuneable laser \#1. TL2: Tuneable laser \#2. Label Rx: Label receiver. Payload Rx: Payload receiver.

such as demultiplexing [13]-[15], wavelength conversion [14], [16]-[18] and all-optical regeneration [19]-[22].

In this paper we report, for the first time to the best of our knowledge, on a novel optical label encoding scheme employing EAM-based wavelength conversion. The two-level labeled signals consist of a $10 \mathrm{~Gb} / \mathrm{s}$ ASK payload and a $2.5 \mathrm{~Gb} / \mathrm{s}$ DPSK label. Through wavelength conversion based on an EAM, the payload is duplicated on a new wavelength, which in advance has been phase-modulated with a new DPSK label, while the old labels, i.e., the original wavelength and the DPSK data on the original wavelength, are completely dropped off. Our experiment also provides a simple approach to evaluate the phase distortion and thus the frequency chirp of the converted signal. By comparing the experimental data with simulated results using a simple model for the chirp, we arrive at an estimate for the chirp $\alpha$-parameter. The influence of dynamic changes of the $\alpha$-parameter is analyzed.

This paper is organized as follows. Experimental set-up and results are given in Section II. A chirp model of EAMs is presented and used to analyze its impact on the phase detection in Section III. In Section IV, we compare the wavelength conversion characteristics of an EAM and an SOA, and possible advantages of the EAM are discussed. Conclusions and acknowledgment are given in Section V and VI, respectively.

\section{EXPERIMENTAL PROCEDURES AND RESULTS}

The experimental set-up is shown in Fig. 1. A continuous-wave $(\mathrm{CW})$ light beam generated by tuneable laser \#1 (TL1) working at $1550 \mathrm{~nm}$ is intensity-modulated by a Mach-Zehnder (MZ) interferometer with a PRBS pattern length of $2^{9}-1$ forming a nonreturn-to-zero (NRZ) signal at 

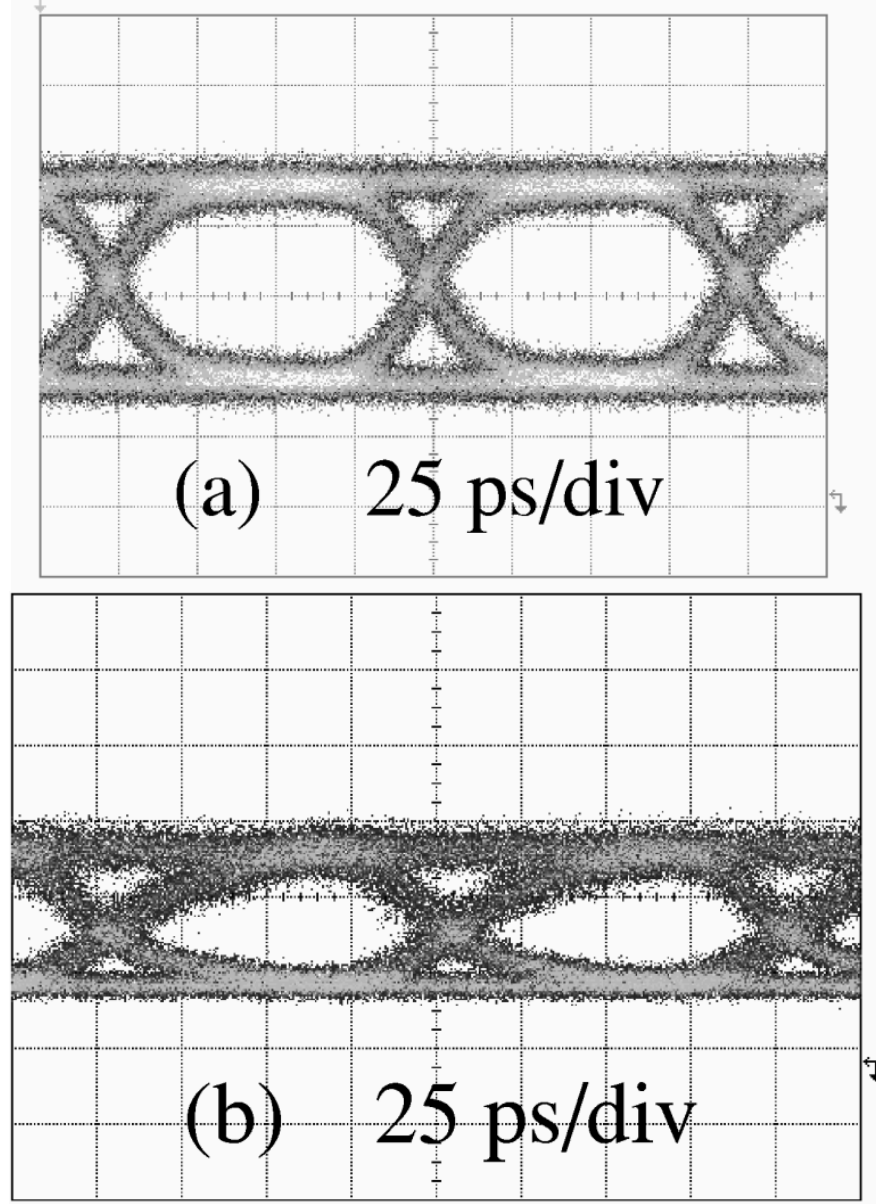

Fig. 2. Eye diagrams of the ASK payload at $10 \mathrm{~Gb} / \mathrm{s}$ of (a) the original signal and (b) the converted signal.

$10 \mathrm{~Gb} / \mathrm{s}$ that serves as the pump, hereafter referred to as an amplitude shift keyed (ASK) signal. Wavelength conversion is performed through cross-absorption modulation (XAM) induced by the pump beam on a probe signal. The probe beam is generated by tuneable laser \#2 (TL2) working at $1555 \mathrm{~nm}$ and is phase-modulated at $2.5 \mathrm{~Gb} / \mathrm{s}$ with a pseudorandom bit sequence (PRBS) pattern length of $2^{7}-1$, hereafter referred to as a differential-phase-shift-keyed (DPSK) signal. Parasitic phase modulation in the EAM, due to refractive index changes induced by the ASK-pump signal, will thus affect the quality of the DPSK signal. The optical power of the pump and probe beams are 20 and $10 \mathrm{dBm}$, respectively. The reverse bias of the EAM is $-2.4 \mathrm{~V}$. After wavelength conversion, the probe beam is filtered out through an optical filter with a bandwidth of $1.6 \mathrm{~nm}$. In the case of conversion to the same wavelength, counterpropagation of the pump and probe can be used to separate the original and the converted signals. Using a $3 \mathrm{~dB}$ coupler, the converted signal is divided into two arms, one for intensity detection and the other for phase detection. The phase detector is a fiber-based delay interferometer (DI) consisting of two 3-dB couplers, which converts the DPSK modulation as well as the frequency chirp into intensity variation. One arm of the interferometer is $8 \mathrm{~cm}$ longer than the other, corresponding to an extra time delay of $400 \mathrm{ps}$. The transmission loss differ-

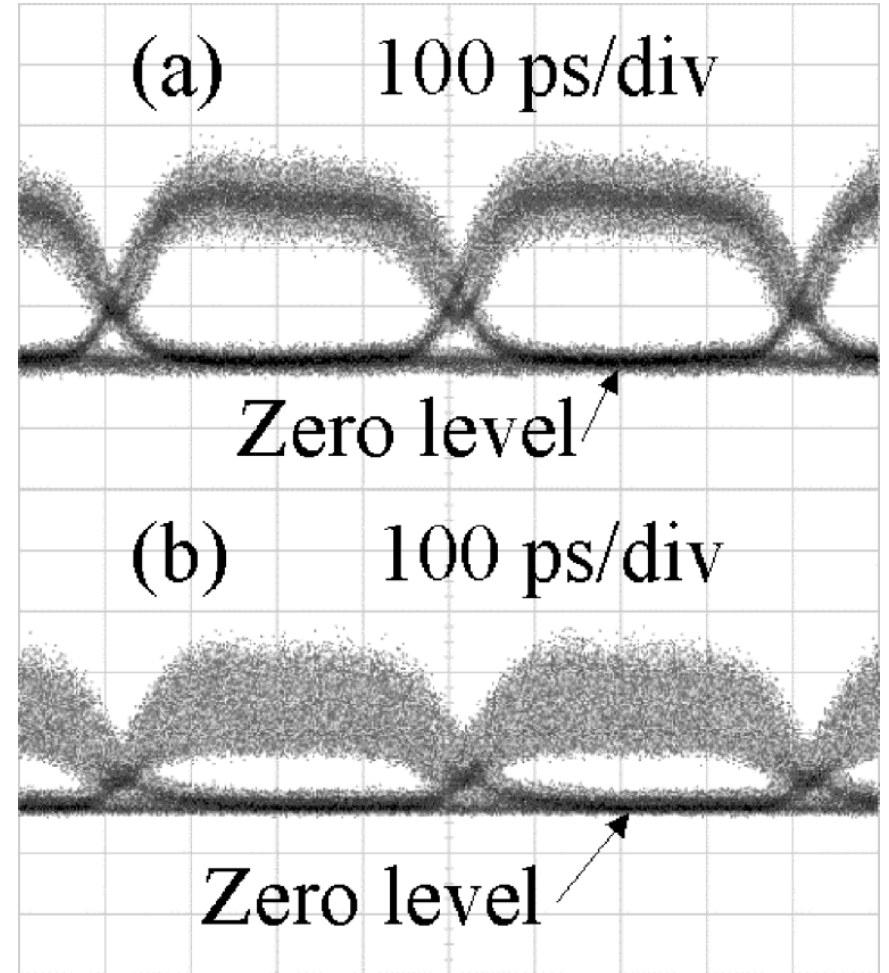

Fig. 3. Eye diagrams of the DPSK label at $2.5 \mathrm{~Gb} / \mathrm{s}$ of (a) the original signal and (b) the converted signal.

ence between the two arms is very small $(\sim 0.17 \mathrm{~dB})$, therefore no loss imbalance effect is observed on the DPSK detection.

The EAMs used in this paper are multiple-quantum-well (MQW) devices with 10-15 quantum wells. The fiber-to-fiber loss at zero bias is about $16 \mathrm{~dB}$ for an uncoated device.

Fig. 2 depicts the ASK eye diagrams of the original signal and the converted signal, clearly showing that the ASK information is successfully duplicated onto the probe beam. Since both mark bits and space bits of the wavelength converted signal carry phase information, the power level of space bits should not be too low to facilitate detection of the phase variation. Therefore a relatively low extinction ratio (ER) of the converted signal is required. Theoretically, an ER up to $9.5 \mathrm{~dB}$ is allowed for DPSK detection. In our experiment, however, we adjust the ER to be about $3.5 \mathrm{~dB}$ because the fiber-based DI is temperature and polarization sensitive and therefore difficult to optimize. Compared to a normal ASK signal with an ER of $10 \mathrm{~dB}$, the power penalty due to low ER is measured to be $\sim 6 \mathrm{~dB}$. The ER of the ASK signal can be improved by using a high-stability DPSK detector and/or a balanced receiver.

The DPSK eye diagrams before and after wavelength conversion are shown in Fig. 3; open eyes of the converted signal are observed. As expected, however, the upper eyelids are broadened considerably while the lower eyelids get only slightly thicker. This can be attributed to the 256 different combinations of ASK bits in one DPSK bit-time of the two arms of the DI, as will be discussed in the following section.

BER measurements as shown in Fig. 4 confirm that the ASK and DPSK components of the converted signal can both have BERs as low as $10^{-9}$, and optical label encoding/recognition is 


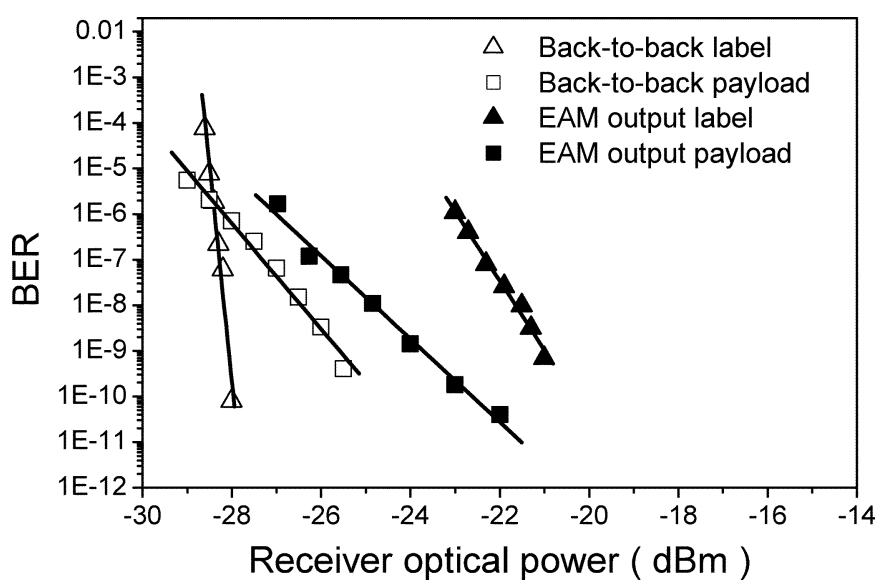

Fig. 4. BER of the payload and the label before and after wavelength conversion.

thus successfully realized. The converted signal is then transmitted over either 50-km SMF or 80-km NZDSF; in both cases error-free operation was obtained for the payload as well as the label.

\section{PUlse ChIRP ANALYSIS}

In this section the influence of the frequency chirp of the wavelength converted signal on the DPSK demodulation will be discussed. Following this discussion, our experimental results will be used to evaluate the chirp.

In the delay interferometer one arm provides an extra delay of $400 \mathrm{ps}$ to the signal, i.e. one bit-time of the DPSK signal, before it is combined with the other arm at the output. The interference between the two arms is generally governed by

$$
\begin{aligned}
E & =P_{A}^{\frac{1}{2}} e^{j\left(\omega t+\varphi_{1}\right)}+P_{B}^{\frac{1}{2}} e^{j\left(\omega t+\varphi_{2}\right)} \\
P & =|E|^{2}=P_{A}+P_{B}+2 \sqrt{P_{A} P_{B}} \cos \left(\varphi_{1}-\varphi_{2}\right) \\
& =P_{A}+P_{B}+2 \sqrt{P_{A} P_{B}} \cos \left(\Delta \varphi_{\text {dpsk }}+\Delta \varphi_{\text {chirp }}\right)
\end{aligned}
$$

where $E$ denotes the output electric field, $P$ the compound optical power, and $\omega$ the optical angular frequency. $P_{\mathrm{A}}$ and $\varphi_{1}$ are the optical power and the phase of one arm, $P_{\mathrm{B}}$ and $\varphi_{2}$ of the other arm. $\Delta \varphi_{\mathrm{dpsk}}$ is the phase difference between the two arms induced by the original DPSK modulation and $\Delta \varphi_{\text {chirp }}$ is the phase difference caused by chirp originating from wavelength conversion (i.e., XAM in the EAM).

In one DPSK bit-time, four ASK bits of one arm overlap with their counterpart in the other arm, resulting in a total of 256 combinations. This is understandable because each arm has $2^{4}(=16)$ combinations of ASK bits and a direct calculation leads to $16 \times 16=256$. However, some combinations have the same consequence, e.g., (1100:0011) and (0011:1100) make no difference, this leads to a dividing factor of 2. Since the DPSK modulation results in a zero or $\pi$ phase difference between the two arms, they may interfere constructively or destructively, resulting in a multiplicative factor of 2 . These two factors counteract each other leading to a total number of 256.

If a photodiode with a bandwidth of $2.5 \mathrm{GHz}$ is used for the detection of the DPSK label, power levels averaged within
TABLE I

\begin{tabular}{|c|c|c|c|}
\hline \multicolumn{2}{|c|}{ ASK Bits } & \multirow{2}{*}{ Power } & \multirow{2}{*}{ Level } \\
\hline Arm 1 & Arm 2 & & \\
\hline \multicolumn{4}{|c|}{ O PSK phase shift between two arms: } \\
\hline 1 & 1 & $P_{1}$ & One \\
\hline 0 & 1 & $\frac{1}{4} P_{1}+\frac{1}{4} P_{0}+\frac{1}{2} \sqrt{P_{1} P_{0}} \cos \Delta \varphi_{\text {chirp }}$ & Two \\
\hline 0 & 0 & $P_{0}$ & Three \\
\hline 1 & 0 & $\frac{1}{4} P_{1}+\frac{1}{4} P_{0}+\frac{1}{2} \sqrt{P_{1} P_{0}} \cos \Delta \varphi_{\text {chirp }}$ & Two \\
\hline \multicolumn{4}{|c|}{ PSK phase shift between two arms: } \\
\hline 1 & 1 & 0 & Five \\
\hline 1 & 0 & $\frac{1}{4} P_{1}+\frac{1}{4} P_{0}-\frac{1}{2} \sqrt{P_{1} P_{0}} \cos \Delta \varphi_{\text {chirp }}$ & Four \\
\hline 0 & 1 & $\frac{1}{4} P_{1}+\frac{1}{4} P_{0}-\frac{1}{2} \sqrt{P_{1} P_{0}} \cos \Delta \varphi_{\text {chirp }}$ & Four \\
\hline 0 & 0 & 0 & Five \\
\hline
\end{tabular}

The Power LeVels Formed IN DPSK Eye Diagrams DUE TO ASK PULSE INTERFERENCE

one DPSK bit-time will be shown due to the slow response, instead of the subtle ASK structure. The averaged power levels depend on the number of the mark bits involved in the interference of the two arms, but different combinations may have different consequences even with the same number of mark bits. For instance, considering the case of 4 mark bits under constructive interference, three different average power levels result from (1111:0000), (1110:1000) and (1100:1100) according to (2), while all other possibilities of combinations do not generate any new levels. This corresponds to the number of different unordered possibilities for filling $r$ slots by $n$ different elements. The solution for this problem is shown in (3)

$$
A(n, r)=C_{n+r-1}^{r} .
$$

In our case, $r$ is the number of ASK bits in one DPSK bit-time and equals 4 , and $n$ is the number of levels within one ASK bit-time. As will be shown in Table I, $n$ equals 3 for constructive interference and 2 for destructive interference. It is thus found that $C_{6}^{2}(=15)$ power levels result from constructive interference and $C_{5}^{1}(=5)$ power levels from destructive interference.

For the purpose of chirp investigation, however, it is convenient to express the interference on the basis of a single ASK bit. The identification of the various signal levels and the corresponding power are given in Table I. Here $P_{1}$ and $P_{0}$ denote the optical power of " 1 " $b$ and " 0 " $b$ of the ASK signal, respectively. The three higher levels form the upper eyelids and the two smaller levels form the lower eyelid. As seen in Table I only level 2 and level 4 are influenced by chirp-induced phase distortion.

The calculated internal structure of DPSK eyes under zero chirp is shown in Fig. 5 (left), where four ASK bit-times within one DPSK bit-time are depicted. The measured DPSK eye diagrams (right) are also given for a direct comparison. Here, the 


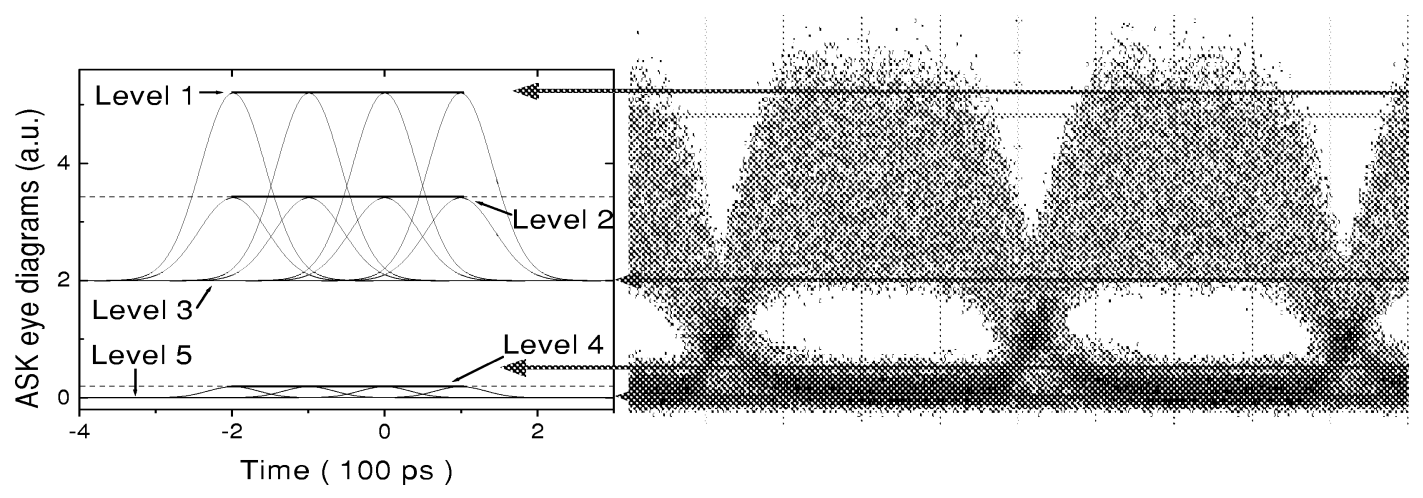

Fig. 5. Simulated DPSK internal structure under zero chirp (left) and experimental results (right).

ER of the ASK signal is taken from experimental data recorded when the DPSK eyes were measured.

As indicated in Table I, five distinct power levels result due to the interference between the two arms of the DPSK demodulator. However, as seen in the right part of Fig. 5, level 2 of the measured DPSK eyes is not distinguished. This is well understood and happens because the DPSK and ASK signals are not synchronized in our set-up. Therefore, only level 4 , as one of the chirp-sensitive levels, can be investigated for comparison purposes. A later discussion will reveal that, compared to level 2 , the impact of level 4 on the DPSK eyes is more important due to its immediate contribution to the closure of the DPSK eyes. Fig. 5 also indicates certain disagreement in level 4 between the left and right parts, suggesting the existence of frequency chirp.

\section{A. Constant Chirp $\alpha$-Parameter}

To gain a basic understanding of how the frequency chirp influences the DPSK demodulation we first employ the assumption of a constant value for the chirp $\alpha$-parameter during an ASK pulse. Equation (4) defines the chirp $\alpha$-parameter according to [23], as follows:

$$
\frac{d \varphi}{d t}=\frac{\alpha}{2 P} \cdot \frac{d P}{d t}
$$

where $\varphi$ and $P$ denote the instantaneous phase and intensity of the output light, respectively.

Since this definition, initially defined for electrical modulation of an EAM, expresses the frequency chirp in terms of optical pulse characteristics (intensity and phase), it can also be used for optical modulation occurring in an EAM, e.g., wavelength conversion.

The simulation results under various constant chirp $\alpha$ values are shown in Fig. 6. It is found that in the presence of chirp, level 4 increases and level 2 decreases, corresponding to an "attraction" of the two chirp-sensitive levels. From this observation it becomes apparent that the change of level 4 is dominant in degrading the DPSK eyes, especially under small frequency chirp [Fig. 6(a)] when level 2 has no contribution at all. From Fig. 6 we find that the DPSK eyes tend to close as the chirp $\alpha$-parameter increases. If the absolute value of $\alpha$ reaches 0.68 , the DPSK eyes become completely closed, as shown in (b). Then it becomes evident that chirp-induced phase distortion is naturally (a)

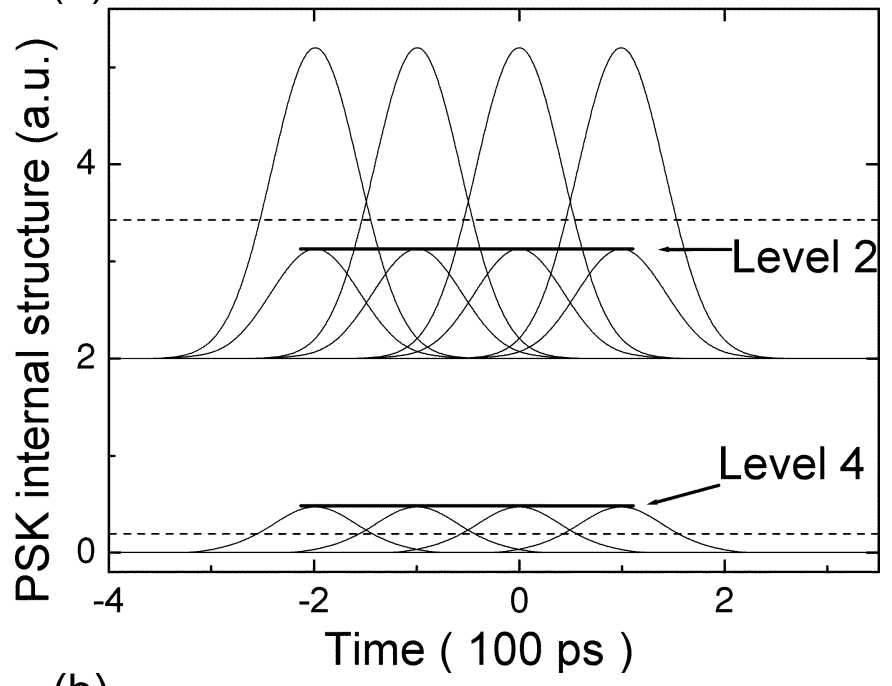

(b)

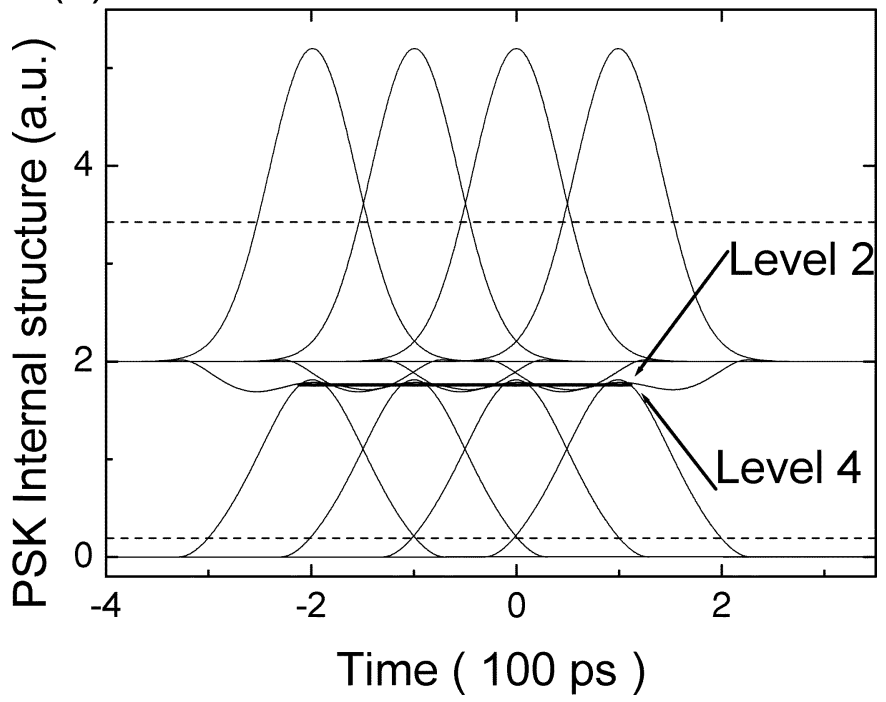

Fig. 6. Simulated DPSK internal structure for different values of the chirp- $\alpha$ parameter: (a) 0.26 and (b) 0.68 . The upper and lower dashed lines indicate levels 2 and 4 in case of no chirp, respectively.

detrimental to DPSK demodulation and will ultimately make the DPSK signal levels indistinguishable; this also means the chirp must be reasonably small $(|a|<0.68)$ if the DPSK eyes are to be clearly open. 


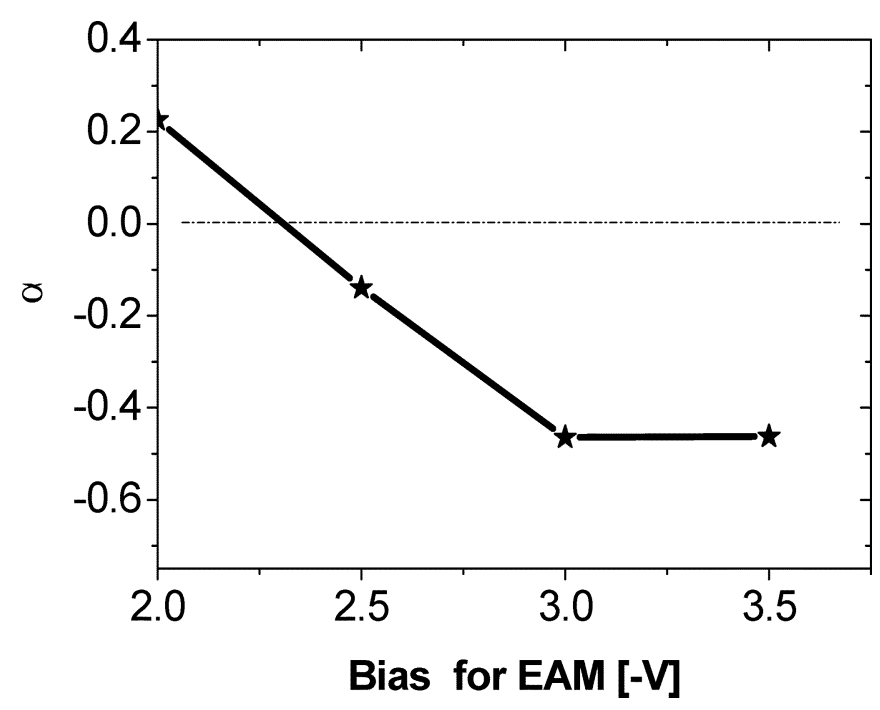

Fig. 7. Measured chirp $\alpha$ versus reverse bias for o/o modulation of EAM.

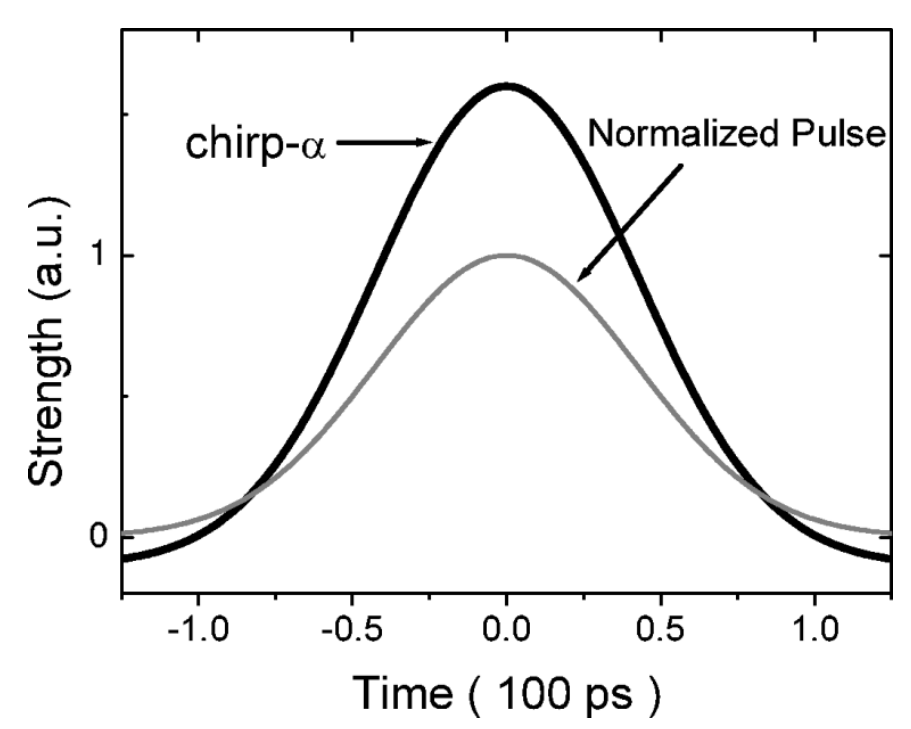

Fig. 8. Chirp $\alpha$ profile for ASK signal.

\section{B. Varying Chirp $\alpha$-Parameter}

Now we consider a more realistic case with the chirp $\alpha$-parameter varying along the ASK pulses. Our earlier measurement of the chirp induced by optical-to-optical (o/o) modulation in an EAM [24], as shown in Fig. 7, suggests a linear variation of the $\alpha$-parameter for reverse voltages lower than $3 \mathrm{~V}$. In this measurement we modified the fiber response method proposed by Devaux [25] by inserting an EAM-based wavelength converter into the transmission line; then the chirp $\alpha$-parameter of the converted signal can be measured with the same principle designed for electrical-to-optical modulation.

If we assume that the change of the effective field seen by the probe beam instantaneously follows the power change of the pump beam, due to the screening effect of pump-photon-generated carriers, then the small signal $\alpha$ will have a profile similar to the pump pulse. This is illustrated in Fig. 8, where the normalized pulse is given for reference. (a)

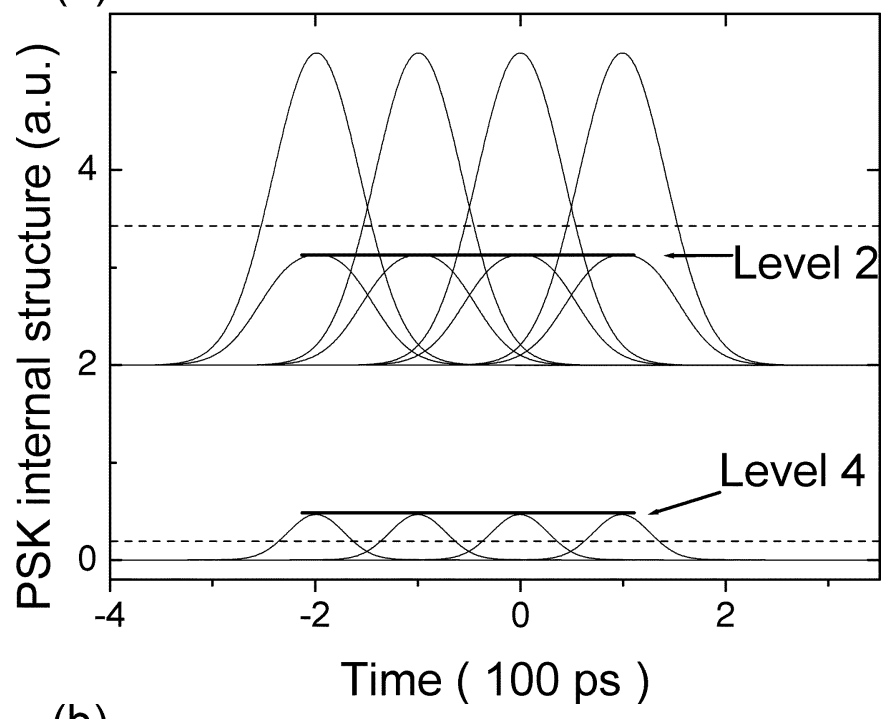

(b)

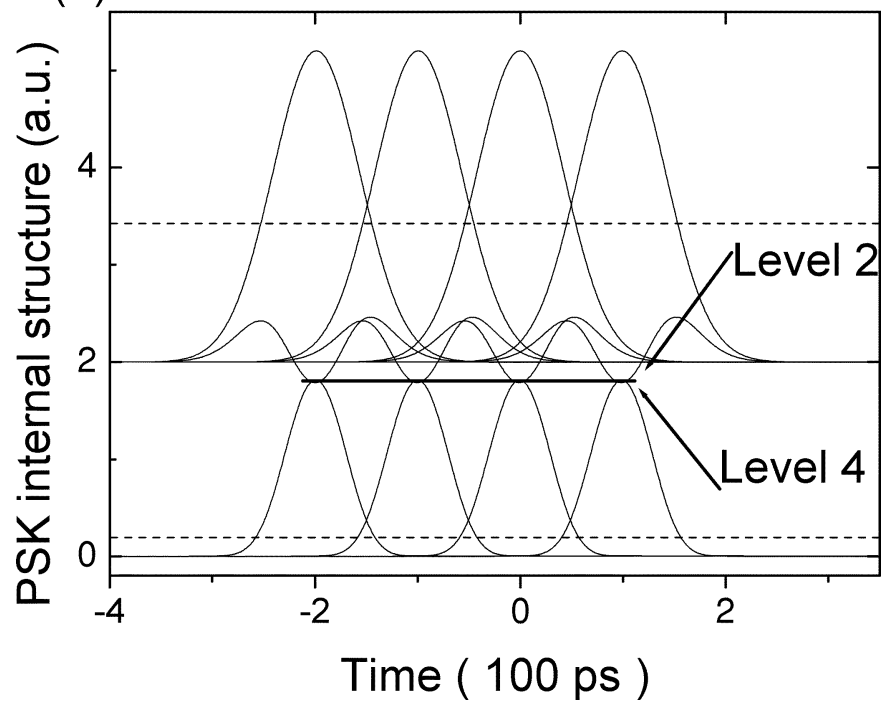

Fig. 9. Internal structure of DPSK eye diagrams, with maximum $\alpha$ of (a) 1.6, (b) 3.6. The upper and lower dashed lines indicate levels 2 and 4 in case of no chirp.

We set the start value of $\alpha$ corresponding to the highest electrical field, i.e., lowest pump power, to be -0.1 . Then with the maximum chirp $\alpha$ as a variable parameter, we calculate the internal structure of the DPSK eye diagrams as shown in Fig. 9. It is found that a much wider dynamic range of chirp $\alpha$ is allowed than for the case of constant $\alpha$ parameter. Thus, as seen in Fig. 9(b), the DPSK eyes completely close for a maximum $\alpha$ of 3.6, whereas in the case of constant $\alpha$ we obtained 0.68 . In case of PRBS where successive " 1 "s or " 0 "s may occur with various pattern length, the chirp $\alpha$-parameters have more complex profiles depending on the patterns. The leading and trailing edges of various patterns still follow the model presented in this sub-section while the flat top regions resulting from more than one mark bit acquire constant chirp $\alpha$ values. However, according to (4), the flat top regions have negligible contribution to the phase change $\left(\Delta \varphi_{\text {chirp }}\right)$ due to the zero amplitude variation $(d P / d t=0)$. Therefore only the two edges of different 
patterns need to be considered, as addressed in the previous discussion.

Our experimental results are compared to this varying chirp $\alpha$-parameter model which applies to an EAM. The fairly good agreement between Fig. 9(a) and the measured DPSK eye diagrams (Fig. 5, right-hand side) suggests an approximate range of chirp $\alpha$ from -0.1 to 1.6 for EAM-based wavelength conversion.

Based on the above discussion, we conclude that phase distortion induced by wavelength conversion may be very harmful to DPSK detection of the converted signal. For a constant value of $\alpha$ a restrictedly small value is required. However, in the case of using an EAM, where $\alpha$ varies dynamically along the wavelength converted pulse, the requirements are relaxed and may be met by a carefully designed EAM.

In addition, when using an EAM as a label encoder (wavelength converter in our scheme), operational parameters like wavelength and reverse bias play an important role in practical cases. With reference to our previous investigation [26], higher pump power (up to $\sim 20 \mathrm{dBm}$ ) and larger reverse bias (up to $\sim 2.5 \mathrm{~V}$ ) are generally desirable in terms of conversion efficiency and frequency chirp. Longer probe wavelengths lead to high conversion efficiency (high extinction ratio) in contrast to shorter wavelengths, while the latter is preferred for low-chirp operation.

\section{Discussion}

Compared to SOAs, which have been used extensively for wavelength conversion through cross gain modulation in the single pass configuration [27], EAMs have several advantages.

First, an EAM allows wavelength conversion with direct data mapping from the pump to the probe beam without any data inversion.

Second, an EAM offers low chirp operation. In our optical label encoding/recognition experiment where attempts were also made to use an SOA for label encoding, we found that wavelength conversion based on an SOA distorted the phase of the probe beam to the extent that it was impossible to detect the DPSK signal.

\section{CONCLUSION}

We have reported on a novel optical label encoding/recognition scheme using EAM-based wavelength conversion, where an orthogonal modulation method is employed for generating the optical packets. We also investigate the chirp properties of the converted signal and analyze its influence on the phase detection. In case of using an EAM as a wavelength converter, we found the phase distortion due to chirp to be relatively small. This is attributed to the variation of $\alpha$ with voltage, allowing relatively high values for the maximum value of $\alpha$.

\section{REFERENCES}

[1] N. Ghani, "Lambda-labeling: a framework for IP-over-WDM using MPLS," Optical Networks Mag., vol. 1, pp. 45-58, 2000.

[2] D. J. Blumental, B. Olsson, and G. Rossi et al., "All-optical label swapping networks and technologies," J. Lightwave Technol., vol. 18, pp. 2058-2075, Dec. 2000.
[3] B. Meagher, G. K. Chang, and G. Ellinas et al., "Design and implementation of ultra-low latency optical label switching for packet-switched WDM networks," J. Lightwave Technol., vol. 18, pp. 1978-1987, Dec. 2000.

[4] M. Hickey, C. Barry, C. Noronha, and L. Kazovsky, "Experimental PSK/ASK transceiver for the STARNET WDM computer communication network," IEEE Photon. Technol. Lett., vol. 5, pp. 568-571, May 1993.

[5] T. Koonen, G. Morthier, J. Jennen, H. Waardt, and P. Demeester, "Optical packet routing in IP-over-WDM networks deploying two-level optical labeling," in Proc. ECOC'01, 2001.

[6] T. Koonen, T. Sulur, I. Monroy, J. Jennen, and H. Waardt, "Optical labeling of packets in IP-over-WDM networks," in Proc. ECOC'02, vol. 5.5.2, 2002.

[7] N. Chi, B. Carlsson, P. V. Holm-Nielsen, C. Peucheret, and P. Jeppesen, "Dispersion management for two-level optically labeled signals in IP-over-WDM networks," in Proc. ECOC'02, vol. 5.5.1, 2002.

[8] N. Chi, B. Carlsson, J. Zhang, P. V. Holm-Nielsen, C. Peucheret, and P. Jeppesen, "Transmission performance of all-optically labeled packets using ASK/DPSK orthogonal modulation," in LEOS 2002, vol. MF3, 2002.

[9] T. H. Wood, "Multiple quantum well (MQW) waveguide modulators," J. Lightwave Technol., vol. 6, pp. 743-757, 1988.

[10] S. Kaneko, M. Noda, Y. Miyazaki, H. Watanabe, and K. Kasahara, "An electroabsorption modulator module for digital and analog applications," J. Lightwave Technol., vol. 17, pp. 669-676, Apr. 1999.

[11] T. H. Wood, J. Z. Pastalan, C. A. Burrus Jr., B. C. Johnson, B. I. Miller, J. L. deMiguel, U. Koren, and M. G. Young, "Electric field screening by photogenerated holes in multiple quantum wells: A new mechanism for absorption saturation," Appl. Phys. Lett., vol. 57, no. 11, pp. 1081-1083, 1990.

[12] M. Suzuki, H. Tanaka, and S. Akiba, "Effect of hole pile-up at heterointerface on modulation voltage in GaInAsP electroabsorption modulators," Electron. Lett., vol. 25, no. 2, pp. 88-89, 1989.

[13] L. K. Oxenløwe, F. Romstad, A. Tersigni, S. Højfeldt, K. Yvind, P. M. W. Skovgaard, K. Hoppe, and J. Hanberg, "Characterization of a MQW electroabsorption modulator as an all-optical demultiplexer," in Proc. LEOS 2001, 2001, Paper ME2.

[14] L. K. Oxenløwe, E. Hilliger, A. Tersigni, A. M. Nik, S. Højfeldt, K. Yvind, P. M. W. Skovgaard, K. Hoppe, and J. Hanberg, "All-optical demultiplexing and wavelength conversion in an electroabsorption modulator," in Proc. ECOC 2001, 2001, Paper Th.B.2.5.

[15] K. Shimomura, K. Shimizu, K. Ishida, T. Sugihara, and Y. Kobayashi, "A simple optical demultiplexing method using saturable absorption of EA modulator," in Proc. ECOC 2000, vol. 3, 2000, Paper 9.4.5, pp. 305-307.

[16] M. Hayashi, H. Tanaka, K. Ohara, T. Otani, and M. Suzuki, "OTDM transmitter using WDM-TDM conversion with an electroabsorption wavelength converter," J. Lightwave Technol., vol. 20, pp. 236-242, Feb. 2002.

[17] L. K. Oxenløwe, A. T. Clausen, and H. N. Poulsen, "Wavelength conversion in an electroabsorption modulator," in Proc. ECOC 2000, 2000, Paper 9.4.4.

[18] N. Edagawa, M. Suzuki, and S. Yamanoto, "Novel wavelength converter using an electroabsorption modulator," IEICE Trans. Electronics, vol. E81-C, no. 8, pp. 1251-2157, 1998.

[19] E. S. Awad, P. S. Cho, C. Richardson, N. Moulton, and J. Goldhar, "Optical $3 R$ regeneration with all-optical timing extraction and simultaneous wavelength conversion using a single electro-absorption modulator," in Proc. ECOC 2002, 2002, Paper 6.3.2.

[20] T. Otani, T. Miyazaki, and S. Yamamoto, " $40-\mathrm{Gb} / \mathrm{s}$ optical $3 \mathrm{R}$ regenerator using electroabsorption modulators for optical networks," J. Lightwave Technol., vol. 20, pp. 195-200, Feb. 2002.

[21] P. S. Cho, P. Sinha, D. Mahgerefteh, and G. M. Carter, "All-optical regeneration at the receiver of $10-\mathrm{Gb} / \mathrm{s} \mathrm{RZ}$ data transmission over 30000 $\mathrm{km}$ using an electroabsorption modulator," IEEE Photon. Technol. Lett., vol. 12, pp. 205-207, Feb. 2000.

[22] S. Højfeldt, S. Bischoff, and J. Mørk, "All-optical wavelength conversion and signal regeneration using an electroabsorption modulator," $J$. Lightwave Technol., vol. 18, pp. 1121-1127, Aug. 2000.

[23] F. Koyama and K. Iga, "Frequency chirping in external modulators," $J$. Lightwave Technol., vol. 6, pp. 87-92, Jan. 1988.

[24] L. Xu, L. K. Oxenløwe, N. Chi, F. P. Romstad, K. Yvind, J. Mørk, P. Jeppesen, K. Hoppe, and J. Hanberg, "Bandwidth and chirp characterization of wavelength conversion based on electroabsorption modulators," in Proc. ECOC 2002, 2002, Paper P1.26.

[25] F. Devaux, Y. Sorel, and J. F. Kerdiles, "Simple measurement of fiber dispersion and of chirp parameter of intensity modulated light emitter," J. Lightwave Technol., vol. 11, pp. 1937-1940, Dec. 1993. 
[26] L. Xu, L. K. Oxenløwe, N. Chi, J. Mørk, P. Jeppesen, K. Hoppe, and J. Hanberg, "Experimental characterization of wavelength conversion at $40 \mathrm{~Gb} / \mathrm{s}$ based on electroabsorption modulators," in Proc. LEOS 2002, 2002, Paper MM3.

[27] T. Durhuus, B. Mikkelsen, C. Joergensen, S. L. Danielsen, and K. E. Stubkjaer, "All-optical wavelength conversion by semiconductors optical amplifiers," J. Lightwave Technol., vol. 14, pp. 942-954, June 1996.

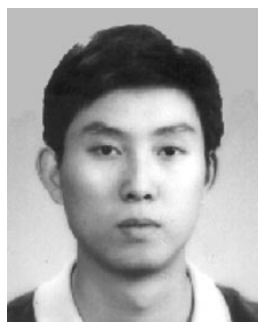

L. Xu was born on January 18, 1975 in Chongqing, China. He received the B.Sc. and M.Sc. degrees in electrical engineering from Beijing University of Posts and Telecommunications, Beijing, China, in 1996 and 1999. He is currently working toward the $\mathrm{Ph} . \mathrm{D}$. degree within the SCOOP project supported by Danish Technical Research Council, at the Research Center COM, Technical University of Denmark (DTU), Lyngby, Denmark.

From 1999 to 2001, he was a submarine cable Project Manager in the International Cable Department of China Telecom. His research interests are concerned with all-optical processing based on semiconductors and system applications of semiconductors.

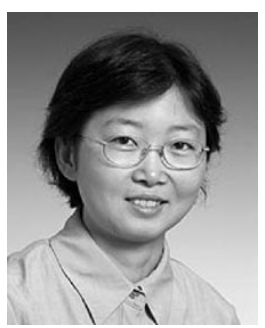

N. Chi was born on March 3, 1974 in Liaoning, China. She received the B.S. and Ph.D. degrees in electrical engineering from Beijing University of Posts and Telecommunications, Beijing, China, in 1996 and 2001, respectively.

Since 2001, she has been an Assistant Professor at the Research Center COM, Technical University of Denmark, Lyngby, Denmark. Her research interests are in the areas of optical packet switching, all-optical processing, and optical labelling.

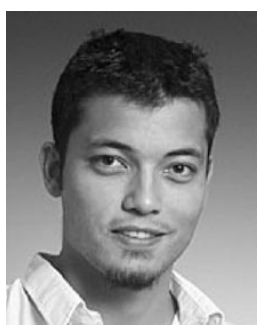

L. K. Oxenløwe received the B.S. degree in physics and astronomy from the Niels Bohr Institute, University of Copenhagen, Copenhagen, Denmark, in 1992. Having studied lasers and optics, he received the International Diploma from Imperial College of Science, Technology and Medicine, London, U.K., and the M.S. degree from the University of Copenhagen in March 1998. He received the Ph.D. degree from COM-Education and Research Center, Technical University of Denmark, Lyngby, in 2002, with work on system characterizations of newly developed devices for optical communications.

$\mathrm{He}$ is currently working on ultrafast communications within the EU IST project TOPRATE.

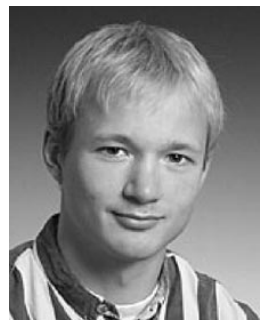

K. Yvind received the M.Sc. degree from the Research Center for Communication, Optics and Materials, the Technical University of Denmark, Lyngby, in 1999 . He is currently working toward the Ph.D. degree.

His work involves design, fabrication, and experiments on InGaAsP optical devices with emphasis on mode-locked lasers, electroabsorption modulators, and semiconductor optical amplifiers for applications in optical communication systems.

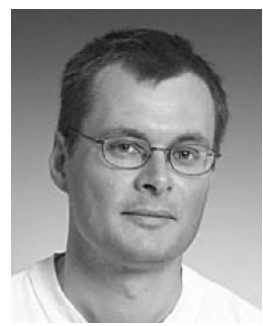

J. Mørk received the M.Sc. and Ph.D. degrees from the Technical University of Denmark, Lyngby, in 1986 and 1988 , respectively.

$\mathrm{He}$ is currrently a Professor in semiconductor devices for optical communication systems and is responsible for modeling and theory in the optoelectronics competence area at the Research Center COM, Technical University of Denmark. His current research interests are concerned with device physics, in particular ultrafast devices for optical signal processing, quantum dot devices, and noise

in nonlinear devices.

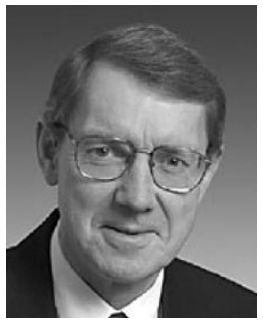

P. Jeppesen (M'69) was born on August 6, 1941 in Vordingborg, Denmark. He received the M.Sc., Ph.D., and Dr.Sc. degrees in electrical engineering from the Technical University of Denmark, Lyngby, in 1967,1970 , and 1978, respectively.

From 1968 to 1969, he was a Research Associate at Cornell University, Ithaca, NY, and from 1969 to 1970, a Project Engineer at Cayuga Associates, Ithaca, NY, both at which he conducted research in the field of GaAs Gunn effect microwave oscillators. From 1970 to 1998, he was an Assistant, Associate, Research, and Full Professor at EMI, Technical University of Denmark, first in microwave electronics and then, since 1974, in optical communications. At EMI, he was Head of the Optogroup from 1974 to 1988 and Head of the Center for Broadband Telecommunications from 1988 to 1998. From 1982 to 1984, he also worked as part-time Manager of R\&D at NKT Elektronik, now Draka Denmark Optical Cable, OFS Fitel Denmark and Tellabs Denmark. From 1995 to 1998 , he coordinated the participation of the Technical University of Denmark in the EU ACTS project METON (METropolitan Optical Network). Since 1999, he has been Professor in optical communications at Research Center COM, Technical University of Denmark, Lyngby, where he is heading the systems competence area. His current research interests are high-speed wavelength-division-multiplexing (WDM) optical communication systems, dispersion maps, WDM devices, and optical signal processing.

Dr. Jeppesen has been a Member of the NATO Research and Technology Board since 1997 and the NATO Science Committee since 1999. He was Chairman of the European Conference on Optical Communication (ECOC) in 1981 and 2002. He received the P. Gorm Petersens Memorial Stipend in 1974, the Esso-prize in 1978, and the Villum Kann Rasmussen Prize in 1988.

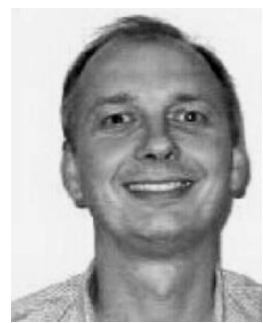

J. Hanberg received the physics and Ph.D. degrees from the University of Copenhagen, Copenhagen, Denmark, in 1983 and 1987, respectively.

$\mathrm{He}$ is currently with Giga-An Intel Company, Skovlunde, Denmark, working with the design, fabrication, and integration of high-speed optoelectronic components. 